\title{
In My Shoes - Validation of a computer assisted approach for interviewing children
}

DOI:

10.1016/j.chiabu.2016.06.022

\section{Document Version}

Accepted author manuscript

Link to publication record in Manchester Research Explorer

\section{Citation for published version (APA):}

Fängström, K., Bokström, P., Dahlberg, A., Calam, R., Lucas, S., \& Sarkadi, A. (2016). In My Shoes - Validation of a computer assisted approach for interviewing children. Child Abuse \& Neglect: the international journal , 58, 160-172. https://doi.org/10.1016/j.chiabu.2016.06.022

\section{Published in:}

Child Abuse \& Neglect: the international journal

\section{Citing this paper}

Please note that where the full-text provided on Manchester Research Explorer is the Author Accepted Manuscript or Proof version this may differ from the final Published version. If citing, it is advised that you check and use the publisher's definitive version.

\section{General rights}

Copyright and moral rights for the publications made accessible in the Research Explorer are retained by the authors and/or other copyright owners and it is a condition of accessing publications that users recognise and abide by the legal requirements associated with these rights.

\section{Takedown policy}

If you believe that this document breaches copyright please refer to the University of Manchester's Takedown Procedures [http://man.ac.uk/04Y6Bo] or contact uml.scholarlycommunications@manchester.ac.uk providing relevant details, so we can investigate your claim.

\section{OPEN ACCESS}


Author accepted manuscript: Fängström, K., Bokström, P., Dahlberg, A., Calam, R., Lucas, S., \& Sarkadi, A. (2016). In My Shoes-Validation of a computer assisted approach for interviewing children. Child Abuse \& Neglect, 58, 160-172.

\section{In My Shoes - Validation of a computer assisted approach for interviewing children}

Karin Fängström ${ }^{\mathrm{a}, \text {, }}$

Pär Bokström ${ }^{\mathrm{a},}$

Anton Dahlberg ${ }^{\mathrm{a}}$

Rachel Calam ${ }^{\text {b, }}$

Steven Lucas ${ }^{\mathrm{a}}$,

Anna Sarkadi ${ }^{\mathrm{a}}$

${ }^{\text {a }}$ Department of Women's and Children's Health, Uppsala University, Islandsgatan 2, 75185 Uppsala, Sweden

${ }^{\mathrm{b}}$ Division of Psychology and Mental Health, School of Health Sciences, The University of Manchester, Second Floor, Zochonis Building, Brunswick Street, Manchester M13 9PL, United Kingdom 


\title{
In My Shoes - Validation of a computer assisted approach for interviewing children
}

\begin{abstract}
Interviewing young children presents a challenge because they tend to provide incomplete accounts and are easily misled. Therefore there is a need for techniques to improve young children's recall, while maintaining accuracy and increasing completeness.

The computer-assisted interview In My Shoes (IMS) is an aid that potentially offers a way for young children to provide accounts of their experiences. This study examined the validity of IMS, by comparing it with a forensic best practice interview approach using a reallife clinical situation to ensure high ecological validity. Children were randomly assigned to either method and both accuracy and completeness of statements made by 4- and 5-yearolds $(\mathrm{N}=54)$ regarding a video-documented health check-up were assessed.
\end{abstract}

The In My Shoes interviews were as good as best practice interviews on all accuracy measures for both age groups, except for object accuracy that was better in the forensic interview condition. Events description completeness was similar in both interview conditions; however, IMS interviews generated more complete statements about people present at the visit. The findings suggest that the IMS approach yields comparable results to a best practice interview, and it can be used as an alternative aid in child interviews.

Keywords: interviewing aid, child, computer-assisted interview, validity, In My Shoes 
Interviews with children are carried out across a range of contexts and for different reasons, and a variety of interviewing strategies and aids have therefore been developed to meet different needs. The aim of the interviews is most often to explore the child's experiences, thoughts, or opinions in order to gain knowledge about the child's perspective (Clark, 2005; Clavering \& McLaughlin, 2010; National Board of Health and Welfare, 2015). In several contexts, such as in social work practice, or in a forensic setting, it is particularly important that reports gathered from children are accurate and extensive and as comprehensive as possible (Aldridge, 1999; Lamb, Orbach, Hershkowitz, Esplin, \& Horowitz, 2007; National Board of Health and Welfare, 2015). This is also true when assessing children's experiences within clinical psychology or a healthcare setting, and the methods employed to do this should bring forward the child's perspective and give reliable and valid information.

Within the forensic context, there is a great deal of empirical research on important components to achieve the goals of acquiring accurate and complete accounts from children. These components includes the types of questions asked by the interviewer, the so called "ground rules," and what is called "narrative practice," i.e., when the child is given a chance to practice narrating events in a particular way.

The type of questions asked is one of the most well researched components of interviewing children, and there is general agreement that open-ended questions are superior to closed-ended ones (Lamb et al., 2007; Lamb et al., 2003). Open-ended questions, such as “Tell me all about..." increase productivity and minimize suggestibility (National Board of Health and Welfare, 2015; Steward et al., 1996), while closed-ended questions, such as a forced choice or multiple choice questions increase the risk of children making errors. The choice of questions is particularly important when interviewing young children, who are more 
sensitive to suggestive and leading questions, compared to older children (Bruck, Ceci, Francouer, \& Renick, 1995; Cassel, Roebers, \& Bjorklund, 1996; Poole \& Lindsay, 1998).

The instructions given to the child at the beginning of the interview, the ground rules, have also been studied. These rules can vary according to different interview protocols. The most common rules are to instruct the child to say, "I don't know" if they do not know the answer, "I don't understand" if they do not understand the question, to correct interviewer error, "If I say something that's wrong, tell me," and instructions not to guess. In some interview protocols, it is also important that the child promises to tell the truth and to practice the difference between the truth and a lie (Faller, 2015). These rules enable children to know the expectations of the interview, which helps them perform better (Lyon, 2014; Saywitz, Snyder, \& Nathanson, 1999), and are thought to improve the accuracy of the reports (Faller, 2015).

The third component, termed narrative practice, is a way of letting the child practice narrating in response to open-ended questions, using a neutral event for this purpose (Lyon, 2014; National Board of Health and Welfare, 2015). Narrative practice has been demonstrated to increase both the accuracy (Roberts, Lamb, \& Sternberg, 2004) and the productivity of child interviews (Brown et al., 2013).

Several interview protocols incorporate all of these components, including the National Institute of Child Health and Human Development (NICHD) Investigative Interview (Lamb et al., 2007; Orbach et al., 2000), the Ten Step Investigative Interview (Lyon, 2014), and the National Children's Advocacy Center (NCAC) Child Forensic Interview Structure (National Children's Advocacy Center, 2012).

\section{NCAC Child Forensic Interview Structure}

One of the most established forensic interviews is that developed by the National Children's Advocacy Center (NCAC) in the United States. The NCAC Child Forensic 
Interview Structure was designed to gather the greatest amount of reliable information in a child-sensitive, developmentally-appropriate, and legally-defensible manner when conducting forensic interviews with children (National Children's Advocacy Center, 2012). It closely follows the structure of the extensively researched NICHD Forensic Interview Protocol (Faller, 2015; Lamb, La Rooy, Malloy, \& Katz, 2011; National Children's Advocacy Center, 2012), however, it has a more flexible structure, which can be adapted to the needs of individual children and community practices. The NCAC has provided training in forensic interviewing for the past 30 years, and more than 100000 child abuse professionals within the United States and 33 countries have been trained in using the NCAC interview protocol (National Children's Advocacy Center, 2015).

\section{Interviewing Aids}

When using the components included in the best practice interviews, children can provide reliable information about their experiences (Hershkowitz, Lamb, Orbach, Katz, \& Horowitz, 2012). However, younger children tend to provide less information and detail than older children in response to open-ended question (Baker-Ward, Gordon, Ornstein, Larus, \& Clubb, 1993; Nelson \& Fivush, 2004), as their memory retrieval strategies and capacities are still developing (Schwenck, Bjorklund, \& Schneider, 2009). Therefore, younger children may need more scaffolded and focused age appropriate narrative questions (Lamb et al., 2003) and/or cues to aid their memory retrieval (Hamond \& Fivush, 1991).

Several attempts have been made to develop techniques to improve children's recall, while maintaining accuracy and increasing completeness. One such technique is the Narrative Elaboration procedure, where pictorial external cues from predefined categories, for example, participants and settings, are used to remind children to report as much detail as possible (Camparo, Wagner, \& Saywitz, 2001; Saywitz \& Snyder, 1996). When this procedure was tested together with the best practice approach, it helped 4-5-year-old children improve recall, 
without compromising accuracy (Dorado \& Saywitz, 2001). Another technique is to help children to mentally reinstate the context before reporting the information (Memon, Meissner, \& Fraser, 2010). This technique is used within the Cognitive Interview (CI) procedure and has proved to increase the details reported by children, especially in 4 to 6-year-olds (Hershkowitz, Orbach, Lamb, Sternberg, \& Horowitz, 2001), without negatively affecting accuracy. For example in a study by Holliday (2003) investigating whether the Cognitive interview facilitated correct recall in 4-5-year-old and 9-10-year-old children, the mean accuracy rate was $81 \%$ for the younger age group. Another technique is to allow children to draw the event, which increased the amount and accuracy of the information that children reported (Butler, Gross, \& Hayne, 1995; Gross \& Hayne, 1998; Katz \& Hershkowitz, 2010). However, in an experimental study, when drawing was used embedded in the best practice recommendations, it did not aid children in remembering more information after an initial interview, and it decreased the mean accuracy from $98 \%$ to $75 \%$ (Salmon, Pipe, \& Malloy, 2012).

\section{Computers in Interviewing}

There is an increasing interest in computer-assisted approaches to interviewing children (Barrow \& Hannah, 2012; Berger, 2006), however there have also been concerns that aids, such as an interactive computer program, could distract the child from the task or engage the child in fantasy play and through this negatively affect for example, the accuracy of recall (Powell, Wilson, \& Hasty, 2002). On the other hand, using the computer as a medium also seems to have several important benefits: the shared external focus on the computer which reduces the social demands of the interview situation, the possibility for the child to exert control over the pace of the interview, the reduced need for eye contact and that it can facilitate communication (Barrow \& Hannah, 2012; Calam, Cox, Glasgow, Jimmieson, \& Groth Larsen, 2000a; Donohue, Powell, \& Wilson, 1999; Jones \& Selby, 1997; Steward et al., 
1996). The presence of a human interviewer together with a computer, i.e. a computerassisted interview (CAI), is a method similar to a regular interview. The interviewer scaffolds the interview and uses the computer together with the interviewee, which creates a three-way process (interviewer- computer - interviewee). There appear to be few studies investigating CAI with children. For example, Connolly (2005) concluded that CAI elicited fewer statements and less sharing of problems than face-to-face interviews. However, one approach very similar to CAI, the Bubble Dialogue, a computer-mediated role-play, has been shown to enhance communication and for the child to express feelings, thoughts and their perspective on events (Jones, Price, \& Selby, 1998; Jones \& Selby, 1997; O'Neill \& McMahon, 1991).

\section{In My Shoes}

The In My Shoes (IMS) computer assisted interview is an aid developed to interview children about their experiences and emotions in relation to different people in different settings. It was initially designed for use in cases where child abuse was suspected, and the aim was for it to have both forensic and therapeutic value (Calam et al., 2000a).

Development of a computer aid was guided by children's interest in computers and studies demonstrating that a computer could help children share information and build rapport with the interviewer (Black \& Ponirakis, 2000; Steward et al., 1996; Watson, Calam, \& Jimmieson, 2002). The IMS interview is a three-way process between the child, the interviewer, and the computer. The trained interviewer sits together with the child and assists, guides, and interacts with her or him through the interview process.

IMS consists of a series of modules that aim to provide a structured format for the interview, moving from less emotive to potentially more emotive material. The modules comprise images of emotions, people, and settings. Furthermore, the intention is to invoke the memory of emotional experiences through these images and to open up areas of questioning (Calam et al., 2000a; Grasso, Atkinson, \& Jimmieson, 2013). Within this format, the children 
can freely express their responses. Everything typed into the computer and all choices made are automatically saved in a log, which means that both the responses and the scenes created by the child can be printed out and used later on (Calam et al., 2000a). Because IMS was designed to include work with children where abuse was suspected, precautions have been taken in designing the interview to avoid leading or disturbing the child through the images. The interviewer is recommended to use open prompts and more focused prompts when needed.

IMS also contains an audio guide, active in the first modules, which aims to engage the child by asking questions such as: "How old are you?" "What do you call this place?" This guide exists in several languages, including sign language, and it can also be turned off.

In a medical context, IMS has shown to be a useful method for interviewing children about their experiences of pain (Calam, Jimmieson, Cox, Glasgow, \& Groth Larsen, 2000b; Watson et al., 2002). In a previous study, investigating the feasibility of IMS for interviewing preschool aged children about a visit at the Child Health Center, it was found that IMS helped children to describe their healthcare experiences in both detail and depth. They expressed shifts in their emotions during the visit and what had made them apprehensive, such as covering their eyes during the optometric test. It was especially apparent that children highlighted details that were important to them, such as the toys available in the waiting room and the types of band-aids or stickers they got to choose after the visit. The children also interacted with the IMS program and maintained their interest for an extended period of time (Bokström, Fängström, Calam, Lucas, \& Sarkadi, 2016). Furthermore, IMS has been used within a school context, to ask children with autism spectrum disorders (ASD) about how they experience their school environment and relations (Barrow \& Hannah, 2012). In a social work context, IMS was used to aid disabled children, who were awaiting adoption, to express their needs and wishes to their social workers (Cousins \& Simmonds, 2011). IMS has also 
proven to be valuable in forensic settings (Calam et al., 2000a). Thus, IMS has been used in various research and it is also used in different clinical contexts, such as the social services. However, to this date there have been no systematic studies of validity, investigating whether the information gathered using IMS is accurate, or how it performs in comparison with the more established and researched interview protocols.

\section{The Current Study}

IMS is an interviewing aid that potentially offers a way for young children to provide accounts of their experiences. In order to be confident that an approach like this is capturing the child's experiences accurately, it is important to assess its validity. Drawing on the knowledge from forensic interviewing, a study was designed to compare IMS with an established, validated best practice interview.

To establish ecological validity, it is important to use a real-life experience, which the child is likely to remember and which is likely to evoke a range of reactions, while not subjecting the child to any additional stresses. We therefore chose to study children's accounts of a routine health check-up at the Child Health Center (CHC). For children aged 4 and 5 years, this visit comprises specific procedures, such as optometric examination and vaccination, respectively. The child's general health and physical and psychosocial development are also assessed during these visits, which usually last 25-45 minutes. In Sweden, more than 99 percent of parents of children up to age six utilize the services of the

CHC (Wallby, 2012). Because the visit includes both interaction and physical contact, this provides a rich opportunity for the validation of children's reports of different types of experiences.

The aim of the present study was to test the validity of the IMS interview approach by comparing it with a slightly modified version of the NCAC Child Forensic Interview Structure, termed the Standard verbal interview, throughout the paper. To evaluate validity, 
both the accuracy and the completeness of the 4- and 5-year-old children's statements regarding a video-documented clinical situation were assessed.

\section{Method}

\section{Participants}

Children aged 4 and 5 years were recruited at five Child Health Centers in areas with varying socio-demographic characteristics in two larger municipalities in Sweden. There were no exclusion criteria except age. In total, 80 children and parents gave their consent to participate. There was a total dropout of 26 children, of which 11 children were never interviewed and 15 interviews had to be excluded from this study for various reasons (Figure 1); four children changed their mind and did not want to be interviewed, and seven children were never interviewed due to an administrative mistake. Out of the 69 interviews conducted, seven were excluded because the children did not talk about the visit at the $\mathrm{CHC}$, two were excluded because the children were interviewed more than 4 weeks after the visit, and six visits were never recorded due to technical problems. The final sample contained 54 children, including 28 4-year-olds (15 girls, 13 boys) and 26 5-year-olds (13 girls, 13 boys).

The children whose parents chose to participate in the study were mainly from families with highly educated parents born in Sweden (Table 1). The children were randomized to being interviewed with either the IMS interview or the Standard verbal interview using stratified randomization based on age ( 4 or 5 years). To control the balance of the interview method, within each stratum, block randomization with blocks of four was used (Altman \& Bland, 1999). Twenty-eight children were interviewed with IMS (15-4-year-olds and 13- 5year-olds) and 26 children were interviewed using the Standard verbal interview (13- 4-yearolds and 13-5-year-olds). 
The study was approved by the Regional Ethical Review Board in Uppsala, Sweden, \# $2012 / 387$.

\section{Procedure}

Nurses at the five Child Health Centers provided information about the study to all families coming for their 4-5-year-old's annual routine check up. The recruitment took place over a 20-month period between 2013 and 2015.

When the parents had given their consent and the child their assent to the nurse to participate in the study, the health visit was video-recorded by the nurse. Interviews were conducted at the child's preschool 2-4 weeks after the visit. The preschool is seen as a safe and secure place for the children, and was therefore chosen as a suitable site to conduct the interview. Since the interviewer was unknown to the child, a preschool teacher or a parent could be present during the interview. However, they were instructed not to interact or say anything during the interview. The interviewer had not seen the video-recorded visit at the $\mathrm{CHC}$ before the interview. The interviews were video-recorded and conducted by either of two interviewers, one male and one female. Both interviewers had a Master of Science degree in sociology or psychology, and they were both trained and accredited in using the IMS computer assisted interview and were trained in the NCAC child forensic interview structure.

Interviews. The sequences of components of the interviews are presented in Figure 2.

Both interview structures comprised the following components, but in a slightly different order: the interviewer introduced herself or himself in the first stage, acquired the child's assent and showed the child how he or she could signal at will to end the interview. The interviewer explained the ground rules and emphasized the importance of telling the truth. Throughout the interview, the interviewer asked mainly open-ended questions such as "Tell me all about..." or "Then what happened?" Detailed questions such as "What did she look like?" or "You mentioned getting a shot, where was your father when you got the shot?" were 
used when needed to scaffold the interview or acquire more details. Direct questions such as yes/no questions or multiple-choice questions were avoided as much as possible, as were leading questions. When the interview was finished, the child chose a sticker, and the interviewer thanked the child for participating. We placed emphasis on ensuring situational consent from all children. No child was pressured to continue participation and the interviewers made sure to tell the child that there was nothing he or she had done wrong if the interview was prematurely finished. Distressed children had immediate access to one of their carers at the preschool.

Standard Verbal Interview Structure. The Standard verbal interview structure closely followed the NCAC Child Forensic Interview structure; however, as these were not forensic interviews, the structure was adapted to suit the purpose of this study. After the introduction, as a means of building rapport, the interviewer asked the child what she or he liked to play. Thereafter the interviewer explained the ground rules. The information gained in the rapport phase was used in a short narrative practice phase. The interviewer then showed the child two pictures of the $\mathrm{CHC}$, one of the entrance to the $\mathrm{CHC}$ building and one of the entrance to the $\mathrm{CHC}$ waiting room. This is not part of the ordinary NCAC structure, but was done to prompt the child to the event that the interview referred to and was considered the start of the substantive phase of the interview. During the substantive phase, the interviewer asked questions, specified above, about the visit and thereafter, thanked the child for participating when the interview was finished.

IMS Interview Structure. After the introduction and the ground rules, the IMS interview was presented to the child and for the remaining interview, it was used together with the child. As the IMS interview allows for a flexible use of its modules, five out of the ten modules were used (see Appendix for an overview of all the IMS modules, available online only). In Module 1 (Introduction), the child chose a self-representational figure, after 
which Module 2 (Emotions) was used to obtain the child's own vocabulary of feelings using the palette of emotion-faces. In Module 3 (Emotions and Scenes), the child practiced how to use the emotion-faces and the answers were typed into the message boxes. These first three modules are designed to help build rapport between the child and the interviewer and to gain a shared vocabulary for the emotions.

The same pictures of the $\mathrm{CHC}$ as in the Standard verbal interviews were then presented to the child with the same wording. The IMS interviews then continued using module 6 (People), where the child was asked to choose representational figures of persons that were present at the $\mathrm{CHC}$ visit and then asked follow-up questions about these persons. In module 7 (Emotions and People), the emotion-faces were used as a stimulus for talking about the visit at the CHC, for example, "Did you ever feel like this when you were at the CHC?" "Tell me all about what happened then.”

During modules 6 and 7, i.e., the substantive phase, the interviewer asked questions, specified above, about the visit and thereafter, thanked the child for participating after the interview was finished.

\section{Coding}

The interviews were video-recorded and transcribed verbatim, and the questions posed by the interviewer as well as the children's statements were coded.

Interviewer questions. Questions asked in the substantive phase of the interviews by the interviewer, including the computer-presented wording in the IMS interviews, were coded and divided into six categories, following the definition of questions in the NCAC Child Forensic Interview Structure: narrative invitation, focused narrative request, detail, yes/no, multiple choice, or leading question. A seventh category, emotional questions, was created based on the features of IMS. 
Narrative invitation An open prompt serving to aid memory recall (e.g., "Tell me all about...")

Focused narrative

request

An open-ended question focusing on a specific person, object, action, or event.

Detail

Yes/No

Multiple choice

Leading

Emotional
A question beginning with what, where, who, when, or how.

A directive question that directs the child to answer "yes" or "no." A question that offers the child two or more answers to choose from (“Did you sit down, stand up or anything else?”)

A question that suggests the particular answer the interviewer is looking to have confirmed (e.g., "You got a shot at the CHC, right?”)

A question about emotions (e.g., "Did you ever feel...?”)

In the analyses, these seven types of questions were collapsed into five main categories similar to definitions in earlier studies: invitations (narrative invitation and focused narrative request), detail questions (wh-questions), directive questions (yes/no and multiple choice), emotional questions, and leading questions.

Children's statements. The children's statements regarding their visit to the CHC were coded and divided into eight categories similar to the definitions in previous studies. These categories were: people, people description, action, action description, action by, action toward, object, and object description . 
People description

Action

Action by

Action toward

Action description

Object

Object description
An elaborate description of a person (e.g. clothes, color of hair)

Anything that someone said or did during the $\mathrm{CHC}$ visit

The person performing the action

The person toward whom the action was directed

An elaborate description of an action

Any object mentioned by the child that was present during the

$\mathrm{CHC}$ visit

An elaborate description of an object

For the purpose of analyses and to make the categories comparable with previous studies (Gross \& Hayne, 1998; Hershkowitz et al., 2012; Lamb et al., 2003; Steward et al., 1996), the eight categories were united into five main categories: total, people, actions (which include action by and action toward), objects, and descriptions (which include people description, action description, and object description). Each statement was also coded as true positive (the child states something to be the case and the video recording supports the statement) or false positive (child states something to be the case and the video recording refutes the statement).

Furthermore, the people present and the central events from the $\mathrm{CHC}$ visit that were reported by the child were coded into the matrix. Central events were defined as examination procedures directed toward the child. These events included physical examination, vaccine injection, vision or hearing screening, and assessment of physical and psychosocial development (e.g., drawing a picture or talking about images that the nurse is showing to the child). The number of central events that a child had been exposed to differed between the children and was therefore calculated separately for each child. The statements about the 
people and the events were then checked against the video recording of the visit at the $\mathrm{CHC}$ for each child.

Interrater reliability. The coding was performed by two individuals, one of whom had no prior knowledge of what occurred during the child's clinical visit or interviews, and one who conducted the interviews. The coders trained on a separate set of transcripts until they reached satisfactory agreement. An interrater reliability (IRR) analysis was performed to assess the degree to which the coders consistently categorized the interviewers' questions and the information provided by the children during the interview. This was done at the beginning and midway through the study. No prevalence problems were indicated by the marginal distributions of the ratings, suggesting that Cohen's (Cohen, 1960) kappa was an appropriate index of IRR (Di Eugenio \& Glass, 2004) and the cut-off values suggested by Landis and Koch (1977), where $\kappa=0.81-1.00$ indicates almost perfect agreement, was used (Landis \& Koch, 1977). The resulting kappa in the first IRR analysis indicated a near perfect agreement

for both the question and the information coding $(\kappa=0.90$ and 0.98 , respectively). The second IRR test was done to guard against observer drift (Kazdin, 1977) and to further investigate the reliability of the coding procedure, and the results indicated a near perfect agreement again for both the question and the information coding $(\kappa=0.87$ and 0.97 , respectively). Hence, the coding procedure was deemed to be adequate for use in this study.

In the next step, one coder compared each child's statements with the videotape of that child's visit to the CHC to assess the accuracy and completeness of the statements.

\section{Outcome Variables}

To investigate the quality of the interviews and to compare the two interview conditions, the length of the interviews in minutes as well as the total number of questions and type of questions were calculated. 
The total accuracy of the child's statements was gained by dividing the total number of true positive statements with the total number of statements. Accuracy was also calculated for each main content category separately (people, action, object, and description).

Completeness was calculated by dividing the child's statements about the people present with all the people present (supported by video recording), which resulted in People Completeness. To obtain Central Events Completeness, statements about the central events were divided by the number of total central events that could be confirmed by the videorecorded clinic visit.

\section{Results}

\section{Statistical Methods}

The mean values and standard deviations were calculated for the length of the interviews, the total number of interviewer questions, and the proportion of type of questions in the substantive phase of the interviews. T-tests were performed to analyze the differences in the total amount of questions and the type of questions posed in the different interview conditions and between the two interviewers.

Linear regression was used to analyze the relationship between the interview method and accuracy and the interview method and completeness, respectively, in two models. Model 1 investigated the individual effect of the independent variables (interview method, child's age and interviewer) on the outcomes using three separate regressions. In Model 2, the effect of the interview method on the outcome was adjusted for the child's age and for the interviewer. See Table 4 and Table 5 for further details of the models. Interaction analyses were conducted between the independent variables for all the outcome variables. A significance level of $p<.05$ and two tailed analyses were applied throughout. The statistical analyses were performed using IBM SPSS Statistics 22.0. 


\section{Preliminary Analyses}

Length of interviews. Independent sample $t$-tests revealed that the time taken in minutes in the IMS interviews $(M=23.12, S D=5.04)$ was significantly longer than the Standard verbal interviews $(M=14.19, S D=4.91), t(52)=6.59, p<.001$. There was also a significant difference in the length of the substantive phase between the interview conditions $t(52)=4.21, p<.001$, with a longer substantive phase in the IMS interviews $(M=11.95, S D$ $=2.20)$ than in the Standard verbal interviews $(M=8.14, S D=2.89)$. There was proportionally less time spent in the substantive phase in the IMS interviews (51.09\%) compared to the Standard verbal interviews $(57.59 \%), t(52)=3.23, p=.020$. A t-test was done to compare if the length of the interviews was different depending on the interviewer, but no significant differences were revealed, $t(52)=0.32, p=.750$.

Total number of questions. In the substantive phase of the IMS interviews, the interviewer and the computer addressed, on average, $27.96(S D=6.06)$ questions to the children, whereas in the Standard verbal interview there were on average $17.69(S D=6.56)$ questions posed to the children, $t(52)=5.98, p<.001$. There was no significant difference between the two interviewers in the total number of questions asked $t(52)=0.58, p=.560$.

Proportion of types of questions. The proportion of different types of questions posed by the interviewer in each interview condition was also calculated (Table 2). In the Standard verbal interviews, the majority of interviewer questions were comprised of invitations $(64.43 \%)$, and there were only a few leading questions $(0.42 \%)$. This indicates that these interviews adhered to the NCAC recommendations (National Children's Advocacy Center, 2012). There were no significant differences in the proportion of directive $t(52)=1.10, p=$ .278 or leading questions $t(52)=0.92, p=.362$ between the interview conditions. However, the IMS interviews contained proportionately fewer invitations $t(52)=6.72, p<.001$ and significantly more detailed questions $t(52)=1.40, p<.001$ and more emotional questions 
$t(52)=11.39, p<.001$ than the Standard verbal interviews. In the IMS interviews, $55.20 \%$ of the detailed questions were asked directly by the computer, and the high proportion of detailed questions and emotional questions reflect the structure of the IMS interviews. We also performed t-tests to investigate if there were any differences in the proportion of types of question posed by the two interviewers, and these revealed no significant differences.

\section{The Accuracy and Completeness of Children's Statements}

Mean values for accuracy and completeness outcome variables are presented by interview method in Table 3. The means are adjusted for child age and interviewer.

Accuracy. The unadjusted linear regression model, Model 1, (Table 4) demonstrated no significant difference in the Total Accuracy between the IMS interviews and the Standard verbal interviews $(\beta=-0.03, p=.581)$. Adjusting for the child's age and the interviewer (Model 2) did not change the accuracy outcome, in relation to the interview method ( $\beta=$ $-0.02, p=.682$ ). No significant interactions were revealed between the independent variables. When analyzing People Accuracy, Action Accuracy, and Description Accuracy, Model 1 revealed no difference in these outcome measures between the interview methods, age categories, or interviewers (Table 4). Neither did Model 2, when the method was adjusted for the child's age and the interviewer. There were no significant interactions for these independent variables. However, for Object Accuracy, Model 1 demonstrated a significantly lower accuracy in the IMS interviews $(\beta=-0.10, p=.037)$ compared to the Standard verbal interviews, while showing a higher accuracy for children aged 5 years $(\beta=0.12, p=.013)$ compared with children aged 4 years. When adjusting for the child's age and the interviewer (Model 2), the difference in Object Accuracy between the interview methods was still significant $(\beta=-0.10, p=.041)$. The interaction analyses demonstrated no significant interactions. 
Completeness. The unadjusted linear regression model, Model 1 (Table 5), revealed that People Completeness was significantly higher in the IMS interviews compared to the Standard verbal interviews $(\beta=0.11, p=.024)$. However, there were no significant differences between the age categories $(\beta=0.01, p=.783)$. When adjusting for the child's age and the interviewer, the difference between the interview methods was still significant $(\beta=0.11, p=.030)$. No significant interactions were revealed between the independent variables.

There was no significant effect of the interview method on Central Events Completeness (Table 5); however, children aged 5 years had a significantly higher completeness compared with children aged 4 years $(\beta=0.14, p=.033)$, and interviews conducted by interviewer 2 compared to interviewer 1 were also significantly less complete on events $(\beta=-0.14, p=.034)$. Adjusting for the child's age and the interviewer, Model 2, did not change the Central Events Completeness in relation to the interview method. No significant interactions were revealed between the independent variables.

\section{Discussion}

\section{The Accuracy and Completeness of Children's Statements}

This is the first study to validate the IMS computer assisted interview against a best practice interview approach using a clinical situation with high ecological validity as an example. To evaluate the IMS interview's validity, we were interested in both the accuracy and the completeness of the children's statements. The results showed that the IMS interviews were as good as the Standard verbal interviews on almost all accuracy measures, i.e., Total Accuracy, People Accuracy, Action Accuracy, and Description Accuracy. The mean accuracy for all accuracy measures in the IMS interviews was high, which is in line with previous research using aids together with the best practice components, such as ground rules and 
open-ended questions (Dorado \& Saywitz, 2001; Hershkowitz et al., 2001; Holliday, 2003; Memon et al., 2010). The only exception was Object Accuracy, which was significantly better in the Standard verbal interviews where it was very high, 0.98; in addition, even though the difference between the methods was significant, the mean Object Accuracy in the IMS interviews was still 0.88 .

There were no significant differences between 4- and 5-year-olds regarding accuracy outcomes, except for Object Accuracy for which the children aged 5 years performed better. Previous research indicates that children as young as 4 years of age can provide reliable and valid information when interviewed properly (Holliday, 2003; Lamb et al., 2007), and when age differences are seen they concern mainly the amount of information that children can provide. For example, Hershkowitz et al. (2012) investigated finer age differences in patterns of response and found that 3- and 4-year-olds were less capable than 5- and 6-year-olds in providing informative and detailed statements.

Children being interviewed with IMS provided more complete statements about the people present at the $\mathrm{CHC}$ visit compared to children interviewed with the Standard verbal interview. In the IMS interviews, module 6 provided the children with pictures of people of different sizes and ethnicity, and the children were encouraged to choose those who were present at the visit. These pictures might have aided the children in remembering more people present at the visit, which was accomplished without decreasing the accuracy, as demonstrated by the People accuracy measure. These results are in line with previous studies, which have demonstrated that external cues, such as pictures, can aid children in remembering information (Camparo et al., 2001; Dorado \& Saywitz, 2001). The Central Events Completeness was similar and low in both the interview conditions. In general, the children only recounted half of the central events of the visit. Perhaps the events, defined by the researchers to be salient and central, were not perceived as equally important by the 
children. On the other hand, when the children's accounts were studied more in depth in a study reported by Bokström et al. (2016), children provided extensive information about other details, for example toys in the waiting room or gifts and tattoo stickers that they received at the visit. This highlights the importance of being clear about the aim when interviewing children, and whether it is intended to obtain information that is perceived as important from an adult perspective or to gain knowledge about the child's subjective experiences, which can differ from the adult's perceptions (Cousins \& Simmonds, 2011; Schalkers, Dedding, \& Bunders, 2014).

\section{Interview Length and Questions}

The analyses revealed that the IMS interviews were longer in minutes compared to the Standard verbal interviews (mean difference was 9 minutes), and there are several factors within the IMS interview approach that could explain this. One factor is the more extensive rapport-building phase in the IMS interviews, where the child names emotions and practices using the emotion-faces. Another important factor in the IMS interviews is that the pace of the interview should be decided by the child (Grasso et al., 2013), which can explain why these interviews took more time. The substantive phase of the IMS interviews was also longer compared to the same phase in the Standard verbal interviews. Choosing and naming figures from the available palettes, asking about each emotion and whether that emotion occurred during the visit at the $\mathrm{CHC}$ were probably the procedures that caused the substantive part of the IMS interviews to be more time consuming. However, as mentioned previously, using pictures probably also increased the People Completeness in the IMS interviews.

The quality of the Standard verbal interviews was good, with a high proportion of invitations and very few leading questions, indicating that these interviews adhered to the NCAC recommendations. The IMS interviews also contained mostly invitations as well as 
detailed questions, reflecting a high level of quality. These interviews also included emotional questions, which are an important foundation of the IMS structure.

\section{Comparing IMS to the Standard Verbal Interviews}

Results from this study suggest that using an interviewing aid, such as the IMS approach, together with components from forensic best practice interviewing, yields comparable accuracy and completeness compared to the standard verbal interview. The question then remains whether it is possible to determine the circumstances under which interviewing aids, such as IMS, could have a comparative advantage or disadvantage. Although this study did not set out to examine this research question, there are some interesting indications in the material.

There were seven interviews in the study that we had to exclude, as the children never talked about the visit to the CHC. All of these children were interviewed with the Standard verbal interview and were aged 4 years. Although not necessarily the case, one possibility is that the IMS interview provided a structure which aided the 4-year-olds' retrieval efforts, similar to using drawing as a retrieval aid (Katz \& Hershkowitz, 2010). This structure could also have reduced the task requirements of the interview situation (Calam et al., 2000a): the external focus on the computer might help to reduce anxiety, by reducing the stress caused by a one-to-one situation between the child and the interviewer (Barrow \& Hannah, 2012) and thus, allow them to perform better (Gross \& Hayne, 1998). Future research should explore this.

\section{Limitations}

The children in the study came from a homogenous background, with highly educated parents born in Sweden. This is a common problem when recruiting families for research in Sweden. Thus, the results may not be generalizable to other populations, and this warrants 
research with children from low SES backgrounds and with other ethnicity than Swedish. Future studies are also needed to determine if the same results will be found in vulnerable populations, for example traumatized children or children with mental illnesses or disabilities. This would be of importance if IMS were to be used, for example, in a forensic context.

The experiences from the interviews is that the children reacted to the interview situation in different ways, some had no problem to talk to the interviewer or to adjust to the novel situation, while others showed clear signs of discomfort and were quiet and/or withdrew behaviorally. Whether the IMS approach benefits all children or if there are individual characteristics of children for whom the interview situation presents a demanding and uncomfortable situation and thus IMS could represent a better approach, also needs to be investigated.

The final sample contained 54 children, with a total dropout of 26 children. Except for the seven interviews discussed above, the dropouts and excluded interviews did not follow a systematic pattern, and therefore we do not believe that this introduced a bias.

Another limitation was that the interviewers were not actually practicing child forensic interviewers or using a standard verbal interview in routine settings, which could have affected their ability to elicit the information. This was however true for both interview conditions.

\section{Conclusion}

This is the first study to validate the IMS computer assisted interview against a best practice interview approach using a clinical situation with high ecological validity as an example. The results showed that the IMS interviews were as good as the Standard verbal interviews in terms of accuracy and completeness. This study suggests that using an interviewing aid, such as the IMS approach, together with components from forensic best 
practice interviewing, yields results comparable to a gold standard verbal interview approach and can be used as an alternative tool in child interviews. 


\section{References}

Aldridge, N. (1999). Enhancing children's memory through cognitive interviewing: An assessment technique for social work practice. Child and Adolescent Social Work Journal, 16, 101-126. http://dx.doi.org/10.1023/A:1022309423642

Altman, D. G., \& Bland, J. M. (1999). How to randomise. BMJ : British Medical Journal, 319, 703-704. http://dx.doi.org/10.1136/bmj.319.7211.703

Baker-Ward, L., Gordon, B. N., Ornstein, P. A., Larus, D. M., \& Clubb, P. A. (1993). Young children's long-term retention of a pediatric examination. Child Development, 64, 1519-1533. http://dx.doi.org/10.2307/1131550

Barrow, W., \& Hannah, E. F. (2012). Using computer-assisted interviewing to consult with children with autism spectrum disorders: An exploratory study. School Psychology International, 33, 450-464. http://dx.doi.org/10.1177/0143034311429167

Berger, M. (2006). Computer assisted clinical assessment. Child and Adolescent Mental Health, 11, 64-75. http://dx.doi.org/10.1111/j.1475-3588.2006.00394.x

Black, M. M., \& Ponirakis, A. (2000). Computer-administered interviews with children about maltreatment: Methodological, developmental, and ethical issues. Journal of Interpersonal Violence, 15, 682-695. http://dx.doi.org/10.1177/088626000015007002

Bokström, P., Fängström, K., Calam, R., Lucas, S., \& Sarkadi, A. (2016). 'I felt a little bubbly in my tummy' : Eliciting pre - schoolers' accounts of their health visit using a computer - assisted interview method. Child: Care, Health and Development, 42, 87-97. http://dx.doi.org/10.1111/cch.12293

Brown, D. A., Lamb, M. E., Lewis, C., Pipe, M.-E., Orbach, Y., \& Wolfman, M. (2013). The NICHD Investigative Interview Protocol: An analogue study. Journal of Experimental Psychology: Applied, 19, 367-382. http://dx.doi.org/10.1037/a0035143

Bruck, M., Ceci, S. J., Francouer, E., \& Renick, A. (1995). Anatomically detailed dolls do not facilitate preschoolers' reports of a pediatric examination involving genital touching. Journal of Experimental Psychology: Applied, 1, 95-109. http://dx.doi.org/10.1037/1076-898X.1.2.95

Butler, S., Gross, J., \& Hayne, H. (1995). The effect of drawing on memory performance in young children. Developmental Psychology, 31, 597. http://dx.doi.org/10.1037/0012-1649.31.4.597

Calam, R., Cox, A., Glasgow, D., Jimmieson, P., \& Groth Larsen, S. (2000a). Assessment and therapy with children: Can computers help? Clinical Child Psychology and Psychiatry, 5, 329-343. http://dx.doi.org/10.1177/1359104500005003004

Calam, R. M., Jimmieson, P., Cox, A. D., Glasgow, D. V., \& Groth Larsen, S. (2000b). Can computer-based assessment help us understand children's pain? European Journal of Anaesthesiology, 17, 284-288. http://dx.doi.org/10.1097/00003643200005000-00002

Camparo, L. B., Wagner, J. T., \& Saywitz, K. J. (2001). Interviewing children about real and fictitious events: Revisiting the narrative elaboration procedure. Law and Human Behavior, 25, 63-80. http://dx.doi.org/10.1023/A:1005691926064

Cassel, W. S., Roebers, C. E. M., \& Bjorklund, D. F. (1996). Developmental patterns of eyewitness responses to repeated and increasingly suggestive questions. Journal of Experimental Child Psychology, 61, 116-133.

http://dx.doi.org/10.1006/jecp.1996.0008 
Clark, A. (2005). Listening to and involving young children: a review of research and practice. Early Child Development and Care, 175, 489-505. http://dx.doi.org/10.1080/03004430500131288

Clavering, E. K., \& McLaughlin, J. (2010). Children's participation in health research: From objects to agents? Child: Care, Health and Development, 36, 603-611. http://dx.doi.org/10.1111/j.1365-2214.2010.01094.x

Cohen, J. (1960). A coefficient of agreement for nominal scales. Educational and Psychological Measurement, 20, 37-46. http://dx.doi.org/10.1177/001316446002000104

Connolly, P. (2005). Children, assessments and computer-assisted interviewing. Child Abuse Review, 14, 407-414. http://dx.doi.org/10.1002/car.922

Cousins, J., \& Simmonds, J. (2011). Investigating the involvement of disabled children in using In My Shoes as a family-finding tool: A pilot project. Adoption \& Fostering, 35, 4-19. http://dx.doi.org/10.1177/030857591103500402

Di Eugenio, B., \& Glass, M. (2004). The kappa statistic: A second look. Computional Linguistics, 30, 95-101. http://dx.doi.org/10.1162/089120104773633402

Donohue, A., Powell, M. B., \& Wilson, J. C. (1999). The effects of a computerised interview on children's recall of an event. Computers in Human Behavior, 15, 747-761. http://dx.doi.org/10.1016/S0747-5632(99)00045-X

Dorado, J. S., \& Saywitz, K. J. (2001). Interviewing preschoolers from low-and middle-SES communities: A test of the Narrative Elaboration recall improvement technique. Journal of Clinical Child Psychology, 30, 568-580. http://dx.doi.org/10.1207/S15374424JCCP3004 12

Faller, K. (2015). Forty years of forensic interviewing of children suspected of sexual abuse, 1974-2014: Historical benchmarks. Social Sciences, 4, 34-65. http://dx.doi.org/10.3390/socsci4010034

Grasso, F., Atkinson, K., \& Jimmieson, P. (2013). In My Shoes - a computer assisted interview for communicating with children about emotions. Paper presented at the 2013 Humaine Association Conference on Affective Computing and Intelligent Interaction (ACII), Geneva, Switzerland.

Gross, J., \& Hayne, H. (1998). Drawing facilitates children's verbal reports on emotionally laden events. Journal of Experimental Psychology: Applied, 4, 163-179. http://dx.doi.org/10.1037/1076-898x.4.2.163

Hamond, N. R., \& Fivush, R. (1991). Memories of Mickey Mouse: Young children recount their trip to disneyworld. Cognitive Development, 6, 433-448. http://dx.doi.org/10.1016/0885-2014(91)90048-I

Hershkowitz, I., Lamb, M. E., Orbach, Y., Katz, C., \& Horowitz, D. (2012). The development of communicative and narrative skills among preschoolers: Lessons from forensic interviews about child abuse. Child Development, 83, 611-622. http://dx.doi.org/10.1111/j.1467-8624.2011.01704.x

Hershkowitz, I., Orbach, Y., Lamb, M. E., Sternberg, K. J., \& Horowitz, D. (2001). The effects of mental context reinstatement on children's accounts of sexual abuse. Applied Cognitive Psychology, 15, 235-248. http://dx.doi.org/10.1002/acp.699

Holliday, R. E. (2003). The effect of a prior cognitive interview on children's acceptance of misinformation. Applied Cognitive Psychology, 17, 443-457. http://dx.doi.org/10.1002/acp.879

Jones, A., Price, E., \& Selby, C. (1998). Exploring children's responses to interpersonal conflict using bubble dialogue in a mainstream and EBD school. Computers \& Education, 30, 67-74. http://dx.doi.org/10.1016/S0360-1315(97)00081-X 
Jones, A., \& Selby, C. (1997). The use of computers for self-expression and communication. Journal of Computing and Childhood Education, 8, 199-214.

Katz, C., \& Hershkowitz, I. (2010). The effects of drawing on children's accounts of sexual abuse. Child Maltreatment, 15, 171-179. http://dx.doi.org/10.1177/1077559509351742

Kazdin, A. E. (1977). Artifact, bias, and complexity of assessment: the ABCs of reliability. Journal of Applied Behavior Analysis, 10, 141-150. http://dx.doi.org/10.1901/jaba.1977.10-141

Lamb, M. E., La Rooy, D. J., Malloy, L. C., \& Katz, C. (2011). Children's testimony: A handbook of psychological research and forensic practice. Chichester, UK: WileyBlackwell.

Lamb, M. E., Orbach, Y., Hershkowitz, I., Esplin, P. W., \& Horowitz, D. (2007). Structured forensic interview protocols improve the quality and informativeness of investigative interviews with children: A review of research using the NICHD Investigative Interview Protocol. Child Abuse and Neglect, 31, 1201-1231. http://dx.doi.org/10.1016/j.chiabu.2007.03.021

Lamb, M. E., Sternberg, K. J., Orbach, Y., Esplin, P. W., Stewart, H., \& Mitchell, S. (2003). Age differences in young children's responses to open-ended invitations in the course of forensic interviews. Journal of Consulting and Clinical Psychology, 71, 926-934. http://dx.doi.org/10.1037/0022-006X.71.5.926

Landis, J. R., \& Koch, G. G. (1977). The measurement of observer agreement for categorical data. Biometrics, 33, 159-174. http://dx.doi.org/10.2307/2529310

Lyon, T. D. (2014). Interviewing children. Annual Review of Law and Social Science, 10, 73-89. http://dx.doi.org/10.1146/annurev-lawsocsci-110413-030913

Memon, A., Meissner, C. A., \& Fraser, J. (2010). The Cognitive Interview: A meta-analytic review and study space analysis of the past 25 years. Psychology, Public Policy, and Law, 16, 340. http://dx.doi.org/10.1037/a0020518

National Board of Health and Welfare. (2015). Listening to children in foster care Eliciting reliable reports from children: Review of influential factors. http://www.socialstyrelsen.se.

National Children's Advocacy Center. (2012). National Children's Advocacy Center's Child Forensic Interview Structure. Huntsville, Alabama.

National Children's Advocacy Center. (2015). National Children's Advocacy Center: History. Retrieved October 7, 2015, from http://www.nationalcac.org/table/about/history/

Nelson, K., \& Fivush, R. (2004). The emergence of autobiographical memory: A social cultural developmental theory. Psychological Review, 111, 486-511. http://dx.doi.org/10.1037/0033-295X.111.2.486

O'Neill, B., \& McMahon, H. (1991). Opening new windows with bubble dialogue. Computers \& Education, 17, 29-35. http://dx.doi.org/10.1016/0360$\underline{1315(91) 90069-4}$

Orbach, Y., Hershkowitz, I., Lamb, M. E., Sternberg, K. J., Esplin, P. W., \& Horowitz, D. (2000). Assessing the value of structured protocols for forensic interviews of alleged child abuse victims. Child Abuse and Neglect, 24, 733-752. http://dx.doi.org/10.1016/S0145-2134(00)00137-X

Poole, D. A., \& Lindsay, D. S. (1998). Assessing the accuracy of young children's reports: Lessons from the investigation of child sexual abuse. Applied and Preventive Psychology, 7, 1-26. http://dx.doi.org/10.1016/S0962-1849(98)80019-X 
Powell, M. B., Wilson, C. J., \& Hasty, M. K. (2002). Evaluation of the usefulness of 'Marvin'; a computerized assessment tool for investigative interviewers of children. Computers in Human Behavior, 18, 577-592. http://dx.doi.org/10.1016/S0747$\underline{5632(02) 00003-1}$

Roberts, K. P., Lamb, M. E., \& Sternberg, K. J. (2004). The effects of rapport-building style on children's reports of a staged event. Applied Cognitive Psychology, 18, 189-202. http://dx.doi.org/10.1002/acp.957

Salmon, K., Pipe, M.-E., \& Malloy, A. (2012). Do non-verbal aids increase the effectiveness of 'best practice' verbal interview techniques? An experimental study. Applied Cognitive Psychology, 26, 370-380. http://dx.doi.org/10.1002/acp.1835

Saywitz, K. J., \& Snyder, L. (1996). Narrative Elaboration: Test of a new procedure for interviewing children. Journal of Consulting and Clinical Psychology, 64, 13471357. http://dx.doi.org/10.1207/s1532480xads0301 7

Saywitz, K. J., Snyder, L., \& Nathanson, R. (1999). Facilitating the communicative competence of the child witness. Applied Developmental Science, 3, 58-68. http://dx.doi.org/10.1207/s1532480xads0301 7

Schalkers, I., Dedding, C. W. M., \& Bunders, J. F. G. (2014). '[I would like] a place to be alone, other than the toilet' - Children's perspectives on paediatric hospital care in the Netherlands. Health Expectations. http://dx.doi.org/10.1111/hex.12174

Schwenck, C., Bjorklund, D. F., \& Schneider, W. (2009). Developmental and individual differences in young children's use and maintenance of a selective memory strategy. Developmental Psychology, 45, 1034. http://dx.doi.org/10.1037/a0015597

Steward, M. S., Steward, D. S., Farquhar, L., Myers, J. E. B., Reinhart, M., Welker, J., ... Morgan, J. (1996). Interviewing young children about body touch and handling (Vol. 61): Monographs of the Society for Research in Child Development.

Wallby, T. (2012). Lika för alla? Social position och etnicitet som determinanter för amning, föräldrars rökvanor och kontakter med BVC. Uppsala University, Uppsala.

Watson, S., Calam, R., \& Jimmieson, P. (2002). Can computers help in assessing children's postoperative pain? Initial validation of a computer-assisted interview. European Journal of Anaesthesiology 19, 510-516. http://dx.doi.org/10.1017/S0265021502000832 
Table 1.

Socio-demographic variables by interview method $(N=54)$

\begin{tabular}{|c|c|c|c|c|c|}
\hline & & \multicolumn{2}{|c|}{$\begin{array}{l}\text { Standard verbal interviews } \\
(n=26)\end{array}$} & \multicolumn{2}{|c|}{$\begin{array}{l}\text { IMS interviews } \\
(n=28)\end{array}$} \\
\hline & & $n$ & $\%$ & $n$ & $\%$ \\
\hline \multirow[t]{2}{*}{ Child's age } & 4 & 13 & 50.0 & 15 & 53.57 \\
\hline & 5 & 13 & 50.0 & 13 & 46.43 \\
\hline \multirow[t]{2}{*}{ Sex } & Girl & 14 & 53.85 & 15 & 53.57 \\
\hline & Boy & 12 & 46.15 & 13 & 46.43 \\
\hline \multirow{3}{*}{$\begin{array}{l}\text { Parent's level } \\
\text { of education }\end{array}$} & Elementary School & 1 & 3.85 & 0 & 0 \\
\hline & High School & 2 & 7.69 & 5 & 17.86 \\
\hline & University/college & 23 & 88.46 & 23 & 82.14 \\
\hline \multirow{2}{*}{$\begin{array}{l}\text { Parent's } \\
\text { country of birth }\end{array}$} & Sweden & 23 & 88.46 & 25 & 89.29 \\
\hline & Other & 3 & 11.54 & 3 & 10.71 \\
\hline
\end{tabular}

Table 2.

Mean (Standard Deviations) and proportion in percent for the five categories of questions by interview method

\begin{tabular}{|c|c|c|c|c|c|c|}
\hline \multirow{3}{*}{$\begin{array}{l}\text { Type of question } \\
\text { Invitation }\end{array}$} & \multicolumn{3}{|c|}{$\begin{array}{l}\text { Standard verbal interviews } \\
(n=26)\end{array}$} & \multicolumn{3}{|c|}{$\begin{array}{l}\text { IMS interviews } \\
(n=28)\end{array}$} \\
\hline & \multicolumn{2}{|c|}{$\mathrm{M}(\mathrm{SD})$} & \multirow{2}{*}{$\begin{array}{l}\% \\
64.43\end{array}$} & \multicolumn{2}{|c|}{$\mathrm{M}(\mathrm{SD})$} & \multirow{2}{*}{$\frac{\%}{42.99}$} \\
\hline & 11.46 & $(4.43)$ & & 12.07 & $(4.07)$ & \\
\hline Detail & 5.50 & $(2.77)$ & 30.64 & 9.78 & $(4.05)$ & 35.05 \\
\hline Directive & 0.62 & $(0.90)$ & 3.12 & 0.61 & $(0.92)$ & 1.99 \\
\hline Emotional & 0.04 & $(0.20)$ & 1.38 & 5.46 & $(2.27)$ & 19.84 \\
\hline Leading & 0.08 & $(0.27)$ & 0.42 & 0.04 & $(0.19)$ & 0.12 \\
\hline
\end{tabular}

Table 3.

Means adjusted for child age and interviewer for each outcome variable

\begin{tabular}{llll}
\hline Outcome & $\begin{array}{l}\text { Standard verbal interviews } \\
\left(n=26^{*}\right)\end{array}$ & & $\begin{array}{l}\text { IMS interviews } \\
\left(n=28^{*}\right)\end{array}$ \\
\cline { 1 - 2 } \cline { 1 - 1 } Total Accuracy & .89 & .86 \\
People Accuracy & .91 & .90 \\
Action Accuracy & .91 & .86 \\
Object Accuracy & .98 & .88 \\
Description Accuracy & .85 & .87 \\
People Completeness & .88 & .99 \\
Central Events Completeness & .41 & .42 \\
\hline
\end{tabular}

* The $\mathrm{n}$ varied slightly for the different outcomes depending on missing data 
Table 4.

Linear regression models of Accuracy by interview method, child's age and interviewer

Model $1^{\mathrm{a}}$

\begin{tabular}{llll}
\hline & {$[95 \% \mathrm{CI}]$} & $p$ & $R^{2}$
\end{tabular}

Total Accuracy

Interview method Standard verbal ref

$\begin{array}{llllll} & \text { IMS } & -0.03 & {[-0.12,0.07]} & .581 & \\ \text { Age } & 4 & \text { ref } & & & .055 \\ & 5 & 0.08 & {[-0.01,0.17]} & .089 & \\ \text { Interviewer } & 1 & \text { ref } & & & .010 \\ & 2 & 0.03 & {[-0.58,0.12]} & .467 & \end{array}$

People Accuracy

Interview method Standard verbal ref

$\begin{array}{ll} & \text { IMS } \\ \text { Age } & 4 \\ & 5 \\ \text { Interviewer } & 1 \\ & 2\end{array}$

Action Accuracy

Interview method Standard verbal ref

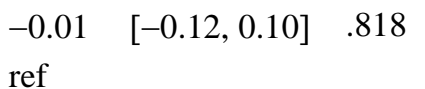

4 ref

$0.07 \quad[-0.03,0.18] \quad .173$

ref

$0.02 \quad[-0.09,0.13] \quad .763$

.001

Model $2^{b}$

\begin{tabular}{llll}
\hline$\beta \quad[95 \% \mathrm{CI}]$ & $p$ & $R^{2}$ \\
\hline & .070
\end{tabular}

.006

$-0.02 \quad[-0.11,0.07] \quad .682$

$0.08 \quad[-0.01,0.17] \quad .091$

$0.03 \quad[-0.06,0.12] \quad .457$

.038 035

002

$0.02 \quad[-0.09,0.13] \quad .740$

.018

$-0.04 \quad[-0.15,0.07] \quad .449$

Age 4

\section{IMS}

4

Interviewer

5

1

2

$$
-0.05
$$$$
[-0.16,0.06] \quad .341
$$

ref

$0.09 \quad[-0.02,0.19] \quad .111$

050

ref $\quad .008$

Object Accuracy

$0.40 \quad[-0.07,0.14] \quad .516$

$0.08 \quad[-0.04,0.19] \quad .124$

$0.03 \quad[-0.08,0.14] \quad .534$

Interview method Standard verbal ref

.089

$$
\text { IMS } \quad-0.10 \quad[-0.20,-0.01] \quad 037
$$$$
-0.10 \quad[-0.19,-0.00] \quad .042
$$

Age 4

ref

5

$0.12 \quad[0.03,0.22] \quad .013$

Interviewer 1

$$
\text { ref } \quad .002
$$

2

$-0.01 \quad[-0.12,0.09] \quad .775$

.002

Description

Accuracy

Interview method Standard verbal ref

$\begin{array}{llllll} & \text { IMS } & 0.02 & {[-0.08,0.10]} & .754 & \\ \text { Age } & 4 & \text { ref } & & & .002 \\ & 5 & 0.01 & {[-0.08,-0.10]} & .748 & \end{array}$

Interviewer $\quad 1$

ref

.000

$\begin{array}{lll}0.12 & {[0.02,0.21] \quad .017}\end{array}$

$-0.01 \quad[-0.11,0.08] \quad .775$

$$
\begin{array}{lllllll}
2 & 0.06 & {[-0.08,0.10]} & .899 & 0.01 & {[-.08,0.10]} & .867
\end{array}
$$

\footnotetext{
${ }^{\mathrm{a}}$ Model 1. Independent variables tested individually on the outcome (measures of Accuracy). $R^{2}$ reported for each independent variable.

${ }^{\mathrm{b}}$ Model 2. Interview method adjusted for age and interviewer. $R^{2}$ reported for the entire Model 2.
} 
Table 5.

Linear regression models of Completeness by interview method, child's age and interviewer

\begin{tabular}{llllll}
\multicolumn{3}{l}{ Model $1^{\mathrm{a}}$} \\
\cline { 2 - 5 }$\beta$ & $\beta$ & {$[95 \%$} & $\mathrm{CI}]$ & $p$ & $R^{2}$ \\
\hline
\end{tabular}

People

Completeness

Interview method Standard verbal ref

$$
\begin{array}{llll}
\text { IMS } & 0.11 \quad[0.02,0.21] & .024
\end{array}
$$

Age

$$
4
$$

5

ref

$0.01 \quad[-0.09,0.11]$

.783

Interviewer

1

2

ref

$-0.03 \quad[-0.13,0.07]$

.517

.094

.001

.008
Model $2^{\mathrm{b}}$

\begin{tabular}{llll}
\hline$\beta$ & {$[95 \% \mathrm{CI}]$} & $p$ & $R^{2}$ \\
\hline
\end{tabular}

.099

$$
0.11 \quad[0.01,0.21] \quad .030
$$

$0.02 \quad[-0.08,0.11] \quad .727$

$-0.02 \quad[-0.12,0.08] \quad .689$

\section{Central Events}

Completeness

.001

Interview method Standard verbal ref

.865

Age

$$
\text { IMS }
$$$$
0.01 \quad[-0.12,0.14]
$$

4 ref

$0.14 \quad[0.01,0.26]$

.033

Interviewer $\quad 1$

ref

$-0.14[-0.26,-0.01] \quad .034$

.084

$0.00 \quad[-0.12,0.12] \quad 986$

$0.13 \quad[0.01,0.25] \quad .035$

.084

2

${ }^{a}$ Model 1. Independent variables tested individually on the outcome (measures of Completeness). $R^{2}$ reported for each independent variable.

${ }^{\mathrm{b}}$ Model 2. Interview method adjusted for age and interviewer. $R^{2}$ reported for the entire Model 2. 


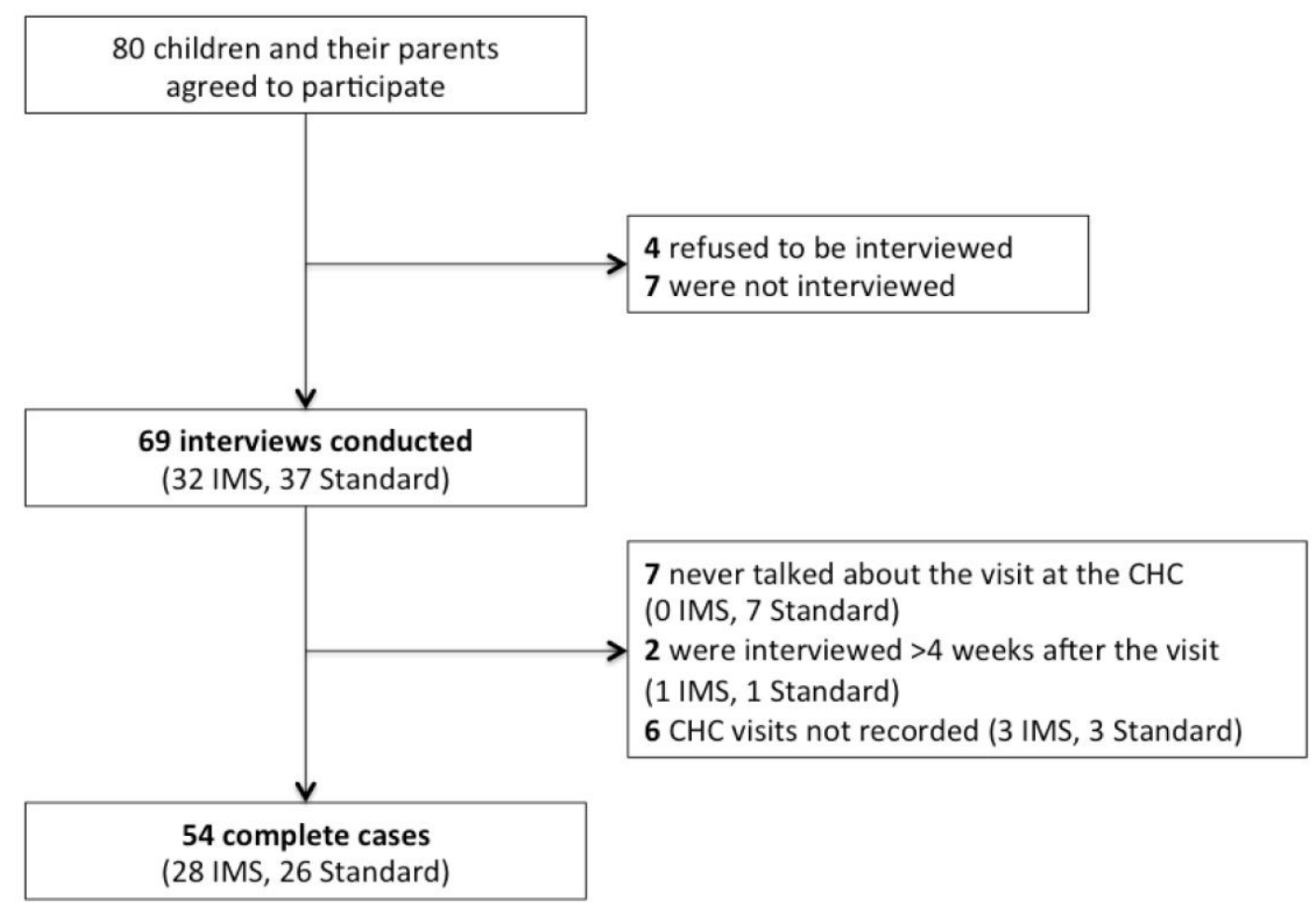

Figure 1. Participant flow chart throughout the recruitment and interviewing process.

\begin{tabular}{|c|c|}
\hline IMS interview structure & Standard verbal interview structure \\
\hline$\downarrow$ & $\downarrow$ \\
\hline \multicolumn{2}{|c|}{$\begin{array}{l}\text { Introduction } \\
\text { Introduce self } \\
\text { Get assent }\end{array}$} \\
\hline$\downarrow$ & $\downarrow$ \\
\hline Explain guidelines/ground rules & Rapport \\
\hline Don't understand & Tell me about some things you like to do/play. \\
\hline Correct me & Explain guidelines/ground rules \\
\hline Don't guess & Don't understand \\
\hline Truth & Correct me \\
\hline Rapport & Don't guess \\
\hline Module One (Introduction) & Truth \\
\hline Module Two (Emotions) & Narrative Practice \\
\hline Module Three (Emotions and Scenes) & Practice narration skills with open-ended question \\
\hline$\downarrow$ & $\downarrow$ \\
\hline \multicolumn{2}{|c|}{ Show pictures of the entrance to the $\mathrm{CHC}$} \\
\hline$\downarrow$ & $\downarrow$ \\
\hline $\begin{array}{l}\text { Substantive Phase } \\
\text { Module Six (People) } \\
\text { Module Seven (Emotions and People) }\end{array}$ & $\begin{array}{l}\text { Substantive Phase } \\
\text { Open-ended prompts and follow-up questions }\end{array}$ \\
\hline$\downarrow$ & $\downarrow$ \\
\hline \multicolumn{2}{|c|}{ Closure } \\
\hline
\end{tabular}

Figure 2. Sequence of the components of the interviews 